\title{
Implementation and Policy Recommendations from the VHA State-of-the-Art Conference on Strategies to Improve Opioid Safety
}

\author{
Joseph W. Frank, MD, MPH ${ }^{1,2} \mathbb{D}$, Amy S. B. Bohnert, PhD, MHS ${ }^{3,4}$, \\ Friedhelm Sandbrink, MD ${ }^{5,6,7}$, Marsden McGuire, $M D, M B A^{8}$, and Karen Drexler, $M D^{8,9,10}$ \\ 'VA Eastern Colorado Health Care System, Aurora, CO, USA; ${ }^{2}$ Division of General Internal Medicine, University of Colorado School of Medicine, \\ Aurora, CO, USA; ${ }^{3}$ University of Michigan Medical School, Ann Arbor, MI, USA; ${ }^{4}$ Center for Clinical Management Research, Veterans Affairs Ann \\ Arbor Healthcare System, Ann Arbor, MI, USA; ${ }^{W}$ Washington DC VA Medical Center, Washington, DC, USA; ${ }^{6}$ Office of Specialty Care Services, \\ Veterans Health Administration, Washington, DC, USA; ${ }^{7}$ George Washington University, Washington, DC, USA; ${ }^{8}$ Office of Mental Health and Suicide \\ Prevention, Veterans Health Administration, Washington, DC, USA; ${ }^{9}$ Atlanta VA Medical Center, Decatur, GA, USA; ${ }^{10}$ Emory University School of \\ Medicine, Atlanta, GA, USA.
}

Evidence-based treatment of opioid use disorder, the prevention of opioid overdose and other opioid-related harms, and safe and effective pain management are priorities for the Veterans Health Administration (VHA). The VHA Office of Health Services Research and Development hosted a State-of-the-Art Conference on "Effective Management of Pain and Addiction: Strategies to Improve Opioid Safety" on September 10-11, 2019. This conference convened a multidisciplinary group to discuss and achieve consensus on a research agenda and on implementation and policy recommendations to improve opioid safety for Veterans. Participants were organized into three workgroups: (1) managing opioid use disorder; (2) Longterm opioid therapy and opioid tapering; (3) managing cooccurring pain and substance use disorder. Here we summarize the implementation and policy recommendations of each workgroup and highlight important cross-cutting issues related to telehealth, care coordination, and stepped care model implementation.

KEY WORDS: opioid use disorder; chronic pain; health policy.

J Gen Intern Med 35(Suppl 3):S983-S7

DOI: $10.1007 / \mathrm{s} 11606-020-06295-\mathrm{y}$

(c) Society ofGeneral InternalMedicine (This is aU.S. government work and not under copyright protection in the U.S.; foreign copyright protection may apply) 2020

\section{INTRODUCTION}

Evidence-based treatment of opioid use disorder (OUD), the prevention of opioid overdose and other opioid-related harms, and the provision of safe and effective acute and chronic pain management are priorities for the Veterans Health Administration (VHA). During a national opioid crisis, Veterans are disproportionately impacted by opioid use disorder and opioid overdose. ${ }^{2}$ Veterans are also more likely to experience chronic pain, posttraumatic stress disorder (PTSD), and suicide than non-Veterans. ${ }^{1,16}$ Efforts to prevent opioid-related harms must prioritize evidence-based strategies to improve opioid safety but must also

Received March 10, 2020

Accepted October 5, 2020

Published online November 3, 2020 ensure access to evidence-based treatments for chronic pain and mental health conditions. As the nation's largest integrated healthcare system, the VHA is ideally suited to address these challenges through broad implementation of evidence-based care and through research to advance the state of the evidence.

\section{METHODS}

To achieve these goals, the VHA Office of Health Services Research and Development (HSR\&D) hosted a State-of-the-Art (SOTA) Conference on "Effective Management of Pain and Addiction: Strategies to Improve Opioid Safety" in September 2019. In preparation for this conference, co-chairs recruited 14 researchers and key clinical stakeholders, both from within and outside VHA, and this group served as the SOTA planning committee. In a planning meeting in January 2019 and a series of follow-up teleconferences, the planning committee achieved consensus on three areas of focus: (1) managing opioid use disorder (OUD); (2) long-term opioid therapy and opioid tapering; (3) managing co-occurring pain and substance use disorder. The planning committee identified subject matter experts for invitation to the SOTA. These invitees were organized into three workgroups, and each workgroup was provided with selected readings and key questions in preparation for the conference.

One of the goals of the conference was to identify areas in which evidence is sufficient for broad implementation and to develop policy recommendations. On the first day of the conference, workgroups sought to prioritize issues that would have the greatest impact for VHA and for Veterans and to achieve consensus, when possible. On the second day, workgroup recommendations were presented to all attendees for discussion and prioritization. Following the conference, recommendations were further refined and prioritized using an iterative discussion among attendees and VHA leadership. In this manuscript, we summarize key implementation and policy recommendations of each workgroup and highlight important cross-cutting themes. 


\section{WORKGROUP RECOMMENDATIONS}

\section{Managing Opioid Use Disorder}

This workgroup focused on improving timely and sustained access to evidence-based medication treatment of OUD (MOUD) by addressing the barriers and facilitators to implementing MOUD. Major barriers to MOUD include: (1) stigma, (2) logistics, (3) treatment experiences and beliefs, and (4) knowledge gaps. ${ }^{14}$ The workgroup's recommendations focused on three main areas: (1) increasing access to MOUD, (2) improving knowledge of MOUD, and (3) improving fidelity to evidence-based models for MOUD (Table 1).

To improve access, the workgroup recommended that every facility be required to provide timely access to MOUD across a range of clinical settings, including Primary Care, Mental Health, and Pain Management settings. Since the conference, several logistical aspects of this recommendation were addressed through a national VHA Notice in October 2019, requiring that facilities confirm removal of barriers to MOUD in all clinical settings and encouraging incentives to facilitate access to MOUD. ${ }^{3}$ The workgroup recommended that psychosocial treatments should be readily available but not mandatory as a prerequisite to receipt of MOUD. To improve access, particularly for rural Veterans who have disproportionately poor access to MOUD, the workgroup recommended increasing support for, and clarifying regulations around, telehealth delivery of MOUD. To increase MOUD access to those at highest risk of overdose death, the workgroup recommended requiring overdose event reporting using the existing Suicide Behavior and Overdose Report electronic health record note template with timely follow-up and engagement in treatment, as is currently required in VHA following a suicide attempt. ${ }^{11}$

Lack of patient and clinician knowledge of MOUD represents another barrier to access. To improve clinician knowledge, the workgroup recommended developing a national Substance Use Disorder (SUD) consultation program based on VHA's successful PTSD and Suicide Prevention consultation services. ${ }^{20}$ The workgroup also recommended broad dissemination of educational resources for both Veterans and VHA healthcare providers. There have been multiple VHA quality improvement initiatives whose goals include educating VHA audiences about MOUD. For example, the VHA's Stepped Care for Opioid Use Disorder Train-the-Trainer (SCOUTT) initiative has been training interdisciplinary teams in Primary Care, Mental Health, and Pain Management to partner with Substance Use Disorder specialty care programs to provide a stepped care model of MOUD treatment.

To implement MOUD with fidelity to the evidence, the workgroup recommended that VHA leadership provide guidance for frequency of monitoring (e.g., urine drug testing) of OUD treatment. For example, for patients initiating MOUD, the group recommended follow-up and urine drug testing at least weekly until negative for 2 weeks, decreasing frequency of monitoring as the patient stabilizes. If opioid abstinence is not achieved within 4
Table 1 Implementation and Policy Recommendations

\begin{tabular}{l}
\hline Goal \\
\hline Managing opioid use disorder \\
1. Increase access to MOUD
\end{tabular}

Specific recommendations

Increase access to MOUD

- Provide same-day access to MOUD at every VHA facility and support medical management of OUD across clinical settings

- Ensure that psychosocial treatment is available but not a pre-requisite for medication treatment

- Clarify regulations around tele-prescribing of MOUD

- Mandate overdose event reporting with the Suicide Behavior and Overdose Report and require timely follow-up following an overdose

2. Improve provider knowledge and comfort to provide MOUD

- Develop national SUD consultation program modeled after existing VHA programs in PTSD and suicide risk management

- Disseminate provider and patient educational resources

- Standardize monitoring of

3. Improve fidelity to evidence-based models of MOUD

substance use during OUD

treatment

- Replace administrative discharges

for substance use with evidence-based treatment of co-occurring SUDs

Long-term opioid therapy and opioid tapering

1. Support evidence-based, patient-centered approaches to opioid therapy and opioid tapering

- Avoid initiation of high-dose longterm opioid therapy for chronic pain - Individualize assessment of risks and benefits and individualize implementation of opioid dose reduction

- Align communication about VHA policy for Veterans, their families/caregivers, healthcare teams and leadership

2. Provide system-wide access to team-based care

- Offer multidisciplinary care with more frequent follow-up during opioid dose reduction

3. Ensure access to assessment $\quad$ Recommend against abrupt and treatment for OUD discontinuation of opioid medications due to concern for OUD without facilitating transition to OUD treatment

Managing co-occurring pain and substance use disorder

1. Improve access to evidence- - Disseminate evidence-based combased non-pharmacologic treatments bined psychological interventions for co-occurring disorders - Disseminate evidence-based interventions for chronic pain (e.g. cognitive behavioral therapy, movement therapies)

MOUD, medications for opioid use disorder; OUD, opioid use disorder; PTSD, post-traumatic stress disorder; SUD, substance use disorder; VHA, Veterans Health Administration

weeks, transition to a higher level of care may be indicated. In addition, the workgroup recommended against discontinuing OUD pharmacotherapy solely for the reason of substance use and recommended increasing care in response by providing evidence-based treatments for cooccurring substance use disorders.

The workgroup's recommendations toward the overarching goal of improving timely and sustained access to MOUD align with other national expert groups. In its 2019 report titled "Medications for Opioid Use Disorder Save Lives", the National Academies of Sciences, 
Engineering, and Medicine recommended that all Food and Drug Administration-approved medications be made available for all people with OUD ${ }^{17}$ It also concluded that "a lack of availability or utilization of behavioral interventions is not a sufficient justification to withhold medications to treat opioid use disorder." Similarly, the 2019 National Drug Control Strategy recommended that federally employed primary care providers should screen for substance use disorders and that SUD treatment should be provided within $24-48$ hours. $^{18}$

\section{Long-term Opioid Therapy and Opioid Tapering}

This workgroup assessed the evidence base for both long-term opioid therapy (LTOT) and for dose reduction and discontinuation of LTOT. ${ }^{7,15}$ The group recommended three goals with specific recommendations for policy and implementation. First, the workgroup recommended that VHA policy support approaches to opioid prescribing that are both evidence-based and patient-centered. There was consensus with the VA and Department of Defense (DoD) Clinical Practice Guideline (CPG) Management of Opioid Therapy for Chronic Pain that (1) clinicians and patients should seek to avoid initiation of long-term, high-dose opioid therapy for patients with chronic pain, and (2) clinicians should provide individualized assessment of risk and benefits of LTOT. The workgroup specifically recommended against policies that interfere with individualized care, and some workgroup members expressed concern about unwarranted variation in opioid-tapering practices between VHA facilities and clinicians. The workgroup members specifically acknowledged potential risks of opioid tapering, in particular with rapid reduction or abrupt discontinuation in patients receiving LTOT. The workgroup recommended clear messaging to Veterans and healthcare providers around VHA policy in support of the VA/DoD CPG for Opioid Therapy regarding the importance of individualized care in LTOT.

Next, the workgroup proposed a goal of providing systemwide access to team-based support for opioid tapering, which has been shown to improve outcomes. ${ }^{4}$ They recommended that multidisciplinary care should be available systemwide, sufficiently staffed to allow timely access by Veterans, and tailored to the needs of individual patients. Thus, the workgroup expressed support for the 2017 VHA mandate of interdisciplinary pain clinics at all VHA facilities, as legislatively required by the Comprehensive Addiction and Recovery Act (CARA) of 2016. Finally, the workgroup proposed a goal of universal access to OUD assessment and treatment, if indicated, for Veterans on LTOT, recommending against discontinuation of LTOT due to concerns for OUD without facilitating transition to evidence-based OUD treatment.

This workgroup's emphasis on individualized, team-based multidisciplinary pain care during both LTOT and opioid tapering is consistent with guidelines from the Centers for Disease Control and Prevention. ${ }^{5,21}$ It is also consistent with recent opioid-tapering guidance from the Centers for Disease Control and Prevention and the Department of Health and Human Services. ${ }^{6,9}$ The workgroup's recommendations sought to target areas where guideline implementation could be enhanced to reduce unwarranted variation and to better support both opioid safety and patient-centered care.

\section{Managing Co-occurring Pain and Substance Use Disorder}

This workgroup discussed challenges in chronic pain management that are unique to individuals with co-occurring SUDs, especially individuals with OUD on long-term MOUD, but also non-opioid SUDs. The workgroup felt that the broader VA initiatives to improve access to evidence-based, nonpharmacologic treatments for chronic pain were particularly important to the population with co-occurring pain and SUD. ${ }^{13}$ They specifically identified two areas in which evidence is sufficient for broad implementation. First, they recommended implementation of combined psychological interventions for co-occurring chronic pain and SUD. For example, for Veterans with pain who are engaged in substance use treatment, a group-based psychological pain management intervention has been shown to reduce pain intensity, reduce alcohol use, and improve pain-related functioning over 12 months. ${ }^{10}$ Addressing both pain and SUD in one program may improve outcomes relative to both in different settings. Although it was developed and tested in specialty addictions treatment, implementation in primary care could be considered. Second, this workgroup recommended ensuring sufficient access to evidence-based non-pharmacologic pain treatment options such as psychological/behavioral therapies (e.g., cognitive behavioral therapy) and movement therapies (e.g., exercise, yoga) for all patients. $^{8}$

\section{Cross-cutting Themes}

The content areas of the three workgroups were designed to be distinct yet complementary, and several cross-cutting themes emerged. First, the VHA consists of a national network of largely urban medical centers and community-based outpatient clinics, many of which provide care for Veterans in rural communities. Nationally, many rural communities lack adequate access to OUD care. ${ }^{19}$ In VHA, in-person care from multidisciplinary teams may not be feasible in some clinics in rural settings. The potential of telehealth-based care to address this important barrier was discussed in all workgroups. The rapid expansion of telehealth in response to the COVID-19 pandemic and changes to federal telehealth policy related to controlled substance prescribing may present new opportunities for telehealth delivery of OUD and chronic pain care, both in VHA and non-VHA healthcare systems. Second, the VHA 
continues to implement a national Community Care Network, in which Veterans can receive care from non-VHA healthcare systems when timeliness or travel distance is a barrier to VHA care. ${ }^{12}$ Workgroups acknowledged the challenge of implementation of evidence-based practices in the context of a rapidly changing landscape of Veterans healthcare delivery. Additional work is needed to understand the impact of this new program on access to care, coordination of care, quality, and cost, especially for vulnerable Veterans with OUD or complex chronic pain and to identify opportunities to partner with non-VHA stakeholders to ensure access to high-quality care in the Community Care Network. Finally, for Veterans receiving care in VHA settings, improved access to OUD and chronic pain care will require improved integration across multiple clinical settings such Primary Care, Mental Health, Pain Management, and other specialty providers. Since 2009, chronic pain care in VHA has been guided by a stepped care model, which calls for population-based screening, assessment, and management of chronic pain using low-intensity interventions in primary care settings with timely access to more intensive treatments for individuals with more complex chronic pain. ${ }^{12}$ Since 2018, the VHA has funded initiatives to implement and evaluate a stepped care model for OUD treatment. Results of these VHA initiatives will likely be relevant to non-VHA integrated healthcare systems seeking to address the same goal of system-wide access to evidence-based OUD and chronic pain care.

\section{CONCLUSION}

Experts participating in the VHA HSR\&D State-of-the-Art Conference provided priority recommendations in three areas: (1) managing opioid use disorder; (2) long-term opioid therapy and opioid tapering; (3) managing co-occurring pain and substance use disorder. Sustained leadership support at the national, regional, and local levels will be necessary to continue progress toward workgroup goals. For many recommendations, additional resources will be required to overcome barriers. Participants recognized the importance of ongoing evaluation of efforts to implement evidence-based practices. With implementation of these recommendations, the Veterans Health Administration can continue to be a national leader in advancing opioid safety.

Acknowledgments: The State-of-the-Art Conference was funded by the Veterans Health Administration Office of Health Services Research and Development. The authors would like to acknowledge the leadership of Dr. David Atkins, Ms. Geraldine McGlynn, Mr. Gerald O'Keefe, and Ms. Karen Bossi in supporting the work of the conference, as well as that of the conference co-chairs, Dr. William Becker and Dr. Keith Humphries, whose intellectual effort in the planning and chairing of the conference is represented in the content of this manuscript.

The views expressed in this manuscript are those of the authors and do not necessarily reflect the position or policy of the Department of Veterans Affairs or the US Government.
Corresponding Author: Joseph W. Frank, MD, MPH; VA Eastern Colorado Health Care System, Aurora, CO, USA (e-mail: joseph. frank@va.gov).

\section{Compliance with Ethical Standards:}

Conflict of Interest: The authors declare that they do not have a conflict of interest.

\section{REFERENCES}

1. 2019 National Veteran Suicide Prevention Annual Report. Washington, DC: Office of Mental Health and Suicide Prevention, U.S. Department of Veterans Affairs; 2019. https://www.mentalhealth.va.gov/docs/datasheets/2019/2019_National_Veteran_Suicide_Prevention_Annual_Report_508.pdf. Accessed 3 March 2020.

2. Bohnert AS, Ilgen MA, Trafton JA, et al. Trends and regional variation in opioid overdose mortality among Veterans Health Administration patients, fiscal year 2001 to 2009. Clin J Pain. 2014;30(7):605-612.

3. Buprenorphine prescribing for opioid use disorder. VHA Notice 2019-18. Washington, DC: Veterans Health Administration, Department of Veterans Affairs; 2019. https://www.va.gov/vhapublications / ViewPublication.asp?pub_ID=8526. Accessed 3 March 2020.

4. Comprehensive Addiction and Recovery Act of 2016. Public Law No. 114198; n.d.. https://www.congress.gov/114/plaws/publ198/PLAW114publ198.pdf. Accessed 29 June 2020.

5. Dowell D, Haegerich TM, Chou R. CDC guideline for prescribing opioids for chronic pain-United States, 2016. JAMA. 2016;315(15):1624-1645.

6. Dowell D, Haegerich T, Chou R. No shortcuts to safer opioid prescribing. N Engl J Med. 2019;380(24):2285-2287.

7. Frank JW, Lovejoy TI, Becker WC, et al. Patient outcomes in dose reduction or discontinuation of long-term opioid therapy: a systematic review. Ann Intern Med. 2017;167(3):181-191.

8. Haffajee RL, Lin LA, Bohnert ASB, Goldstick JE. Characteristics of US counties with high opioid overdose mortality and low capacity to deliver medications for opioid use disorder. JAMA Netw Open. 2019;2(6):e196373.

9. HHS Guide for Clinicians on the Appropriate dosage reduction or discontinuation of long-term opioid analgesics. Washington, DC: U.S. Department of Health and Human Services; 2019. https://www.hhs. gov/opioids/sites/default/files/2019-10/Dosage_Reduction_Discontinuation.pdf. Accessed $14 \mathrm{Feb} 2020$.

10. Ilgen MA, Bohnert AS, Chermack S, et al. A randomized trial of a pain management intervention for adults receiving substance use disorder treatment. Addiction. 2016;111(8):1385-1393.

11. Kemp J, Bossarte RM. Surveillance of suicide and suicide attempts among veterans: addressing a national imperative. Am J Public Health. 2012;102 Suppl 1:e4-5.

12. Kerns RD, Philip EJ, Lee AW, Rosenberger PH. Implementation of the Veterans Health Administration National Pain Management Strategy. Transl Behav Med. 2011;1(4):635-643.

13. Kligler B, Bair MJ, Banerjea R, et al. Clinical policy recommendations from the VHA State-of-the-Art Conference on non-pharmacological approaches to chronic musculoskeletal pain. J Gen Intern Med. 2018;33(Suppl 1):16-23.

14. Mackey K, Veazie S, Anderson J, Bourne D, Peterson K. Evidence brief: Barriers and facilitators to use of medications for opioid use disorder. Washington, DC: Evidence Synthesis Program, Health Services Research and Development Service, Office of Research and Development, Department of Veterans Affairs, 2019. VA ESP Project \#09-009. https:// www.hsrd.research.va.gov/publications/esp/reports.cfm. Accessed 3 March 2020.

15. Mackey K, Anderson J, Bourne D, Chen E, Peterson K. Evidence brief: Benefits and harms of long-term opioid dose reduction or discontinuation in patients with chronic pain. Washington, DC: Evidence Synthesis Program, Health Services Research and Development Service, Office of Research and Development, Department of Veterans Affairs; 2019. VA ESP Project \#09-199. Available at: https://www.hsrd.research.va.gov/ publications/esp/reports.cfm. Accessed 3 Mar 2020.

16. Nahin RL. Severe Pain in Veterans: The effect of age and sex, and comparisons with the general population. J Pain. 2017;18(3):247-254.

17. National Academies of Sciences, Engineering, and Medicine. Medications for Opioid Use Disorder Save Lives. Washington, DC: The National Academies Press. 2019. https://doi.org/10.17226/25310. Accessed 14 Feb 2020 
18. National Drug Control Strategy. Washington, DC: Office of National Drug Control Policy; 2019. https://www.whitehouse.gov/wp-content/uploads/2019/01/NDCS-Final.pdf. Accessed 3 March 2020.

19. Nevedal AL, Wagner TH, Ellerbe LS, Asch SM, Koenig CJ. A qualitative study of primary care providers' experiences with the Veterans Choice Program. J Gen Intern Med. 2019;34(4):598-603.

20. PTSD and suicide: National Center for PTSD Fiscal Year 2018 Annual Report. Washington, DC: National Center for PTSD;2018. https://www. ptsd.va.gov/about/work/docs/annual_reports/2018/NCPTSD_2018_ Annual_Report.pdf. Accessed 3 March 2020.
21. VA/DoD clinical practice guideline for opioid therapy for chronic pain. Department of Veterans Affairs and Department of Defense; 2017. https://www.healthquality.va.gov/guidelines / Pain / cot/ VADoDOTCPG022717.pdf. Accessed 14 Feb 2020.

Publisher's Note: Springer Nature remains neutral with regard to jurisdictional claims in published maps and institutional affiliations. 